\title{
Anorexia nerviosa en adolescentes y adultos.
}

\section{Anorexia nervosa in adolescents and adults.}

Pág. 212,222

Recibido: 04-07-2020

Aceptado: 17-07-2020

Sofía Segreda Castro ${ }^{1}$

Cristine Segura Araya ${ }^{2}$

1. Nutricionista y Medico General.Trabajador Independiente, Costa Rica.

2. Medico General. Trabajador Independiente. San José, Costa Rica

\section{RESUMEN}

La anorexia nerviosa se considera una patología típica de clase social media y alta y se caracteriza por el miedo excesivo a aumentar de peso y/o una alteración en la imagen corporal, que motiva a la persona a implementar una severa restricción dietética u otros comportamientos de pérdida de peso como actividad física excesiva. Esta patología se clasifica en dos tipos: la restrictiva y la de tipo compulsivo purgativo.

El tratamiento de esta enfermedad siempre requiere manejo por parte de un equipo multidisciplinario conformado principalmente por médicos, nutricionistas y psicólogos entrenados en el manejo de trastornos de la conducta alimentaria.

\section{PALABRAS CLAVE}

Índice de Masa Corporal, Trastorno Alimenticio, Anorexia nerviosa.

\section{ABSTRACT}

Anorexia nervosa is considered a typical middle and upper social class pathology and is characterized by excessive fear of gaining weight and/or an alteration in body image, wich motivates the person to implement severe dietary restriction or other behaviors of weight loss as excessive physical activity. This pathology is classified in two types: restrictive and compulsive-purgative.

The treatment of this disease always requires management by a multidisciplinary team consisting mainly of doctors, nutritionists and psychologists trained in the management of eating disorders.

\section{KEY WORDS}

Body Mass Index, Eating Disorder, Anorexia nervosa.

\section{INTRODUCCIÓN}

Los trastornos de conducta alimentaria son un grupo de enfermedades psiquiátricas con presentación más frecuente entre los $13-14$ 
años y alrededor de los 18 años de edad, con repercusiones físicas y psicológicas, originadas y perpetuadas por el mismo individuo, factores socioculturales y familiares. Estas patologías son potencialmente mortales de no ser identificadas y tratadas, por lo que es de suma importancia su detección temprana y el reconocimiento de factores de riesgo como lo son la internalización de una figura ideal delgada, insatisfacción corporal, dietas, afecto negativo, presión percibida sobre delgadez y apoyo familiar carente (1) (2) (3) (4) (5).

Los trastornos alimentarios afectan al 13\% de las mujeres y en cuanto a los hombres no existen datos claros sobre la afectación en ellos, pero se sabe que cada día se diagnostican más casos y que podría no existir diferencia de sexo en cuanto a la edad de presentación (5) (6).

La anorexia nerviosa pertenece a este grupo de trastornos mentales, se considera una patología típica de clase social media y alta y se caracteriza por el miedo excesivo a aumentar de peso y/o una alteración en la imagen corporal, que motiva a la persona a implementar una severa restricción dietética u otros comportamientos de pérdida de peso como actividad física excesiva. Esta enfermedad presenta una alteración marcada en el funcionamiento cognitivo y emocional y puede llegar a desarrollar complicaciones médicas que afectan todos los órganos y sistemas. Además, la anorexia nerviosa, al igual que todos los trastornos de la conducta alimentaria, se encuentra relacionada con comorbilidades psiquiátricas en más del $70 \%$ de las personas, siendo los más frecuentes el abuso de sustancias, los trastornos de ansiedad, del estado de ánimo, del neurodesarrollo y de la personalidad (2) (3).

A pesar de décadas de investigación sobre esta enfermedad, el pronóstico en estos individuos continúa siendo muy pobre. De hecho, en un estudio de cohorte se demostró que solo el $30 \%$ de los pacientes con anorexia nerviosa lograron recuperarse luego de 9 años de tratamiento (7).

\section{DEFINICIÓN}

La anorexia nerviosa se define como la combinación de una ingesta calórica que se encuentra significativamente restringida y el abuso de actividad física, típicamente de inicio en la adolescencia temprana. El individuo presenta alteración de la imagen corporal, miedo al aumento de peso, anosognosia (dificultad que experimenta el sujeto para identificarse como afectado por la enfermedad) y alexitimia (imposibilidad para reconocer y describir las propias emociones). Estos comportamientos se acompañan de conductas obsesivas, que llevan al padecimiento de distintas complicaciones orgánicas como desnutrición y alteraciones hidroelectrolíticas (1) (8) (9) (10) (21) (22).

\section{CLASIFICACIÓN}

Tanto el CIE-10 como el DSM-V utilizan el índice de masa corporal (IMC) bajo como diagnóstico. Además, el DSM-5 usa este parámetro para referirse a la gravedad (12). Se puede subclasificar según su severidad en leve $>17$ $\mathrm{kg} / \mathrm{m}^{2}$, moderado $16-16.99 \mathrm{~kg} / \mathrm{m}^{2}$, severo $15-$ $15.99 \mathrm{~kg} / \mathrm{m}^{2}$ y extremo $<15 \mathrm{~kg} / \mathrm{m}^{2}$ (11). Por otro lado, la anorexia se clasifica en dos tipos, la de tipo restrictivo, que consiste en la disminución severa de la ingesta primordialmente de lípidos y carbohidratos y la de tipo compulsivo purgativo, en la que el paciente presenta restricción de alimentos por períodos extensos seguidos por atracones, para por último compensar con purgas creando así un ciclo repetitivo (10).

Criterios diagnósticos basados en el DSM-V y el CIE-10: 
Dentro de los criterios diagnósticos se pueden encontrar los siguiente:

1. Restricción intencional o evitación de la ingesta calórica en relación con los requerimientos, lo cual altera el IMC, siendo este significativamente bajo para el sexo, edad, etapa de desarrollo y salud física del paciente (13).

2. Miedo intenso a engordar o aumentar de peso, presencia de comportamiento obsesivo que interfiere con la ganancia ponderal, a pesar de que el paciente presente un peso bajo (13).

3. Alteración en la forma en que se percibe el peso o forma de su cuerpo (13).

4. Disfunción de tipo endocrina que causa pérdida de periodos menstruales o retraso en la menarca (13).

\section{CRITERIOS DE HOSPITALIZACIÓN}

El papel del internamiento hospitalario en pacientes anoréxicos ha presentado cambios importantes en las últimas tres décadas. Esto porque previamente se preferían internamientos largos, en los cuales el sujeto era estabilizado y posteriormente se trataba su problema metabólico de base hasta una recuperación completa, la cual con frecuencia incluía tratar el trastorno psiquiátrico. Sin embargo, los altos costo en las estancias hospitalarias han hecho que se prefiera una nueva estrategia de manejo (14).

La táctica de manejo actual se basa en mantener un control ambulatorio del paciente y solamente se hospitalizará al individuo cuando su vida se encuentre en riesgo. Los criterios de internamiento se basan en el contexto de una persona previamente conocida con desorden alimenticio, que se presenta con signos vitales inestables (14).
Dentro de estos criterios se incluyen signos clínicos como: bradicardia, con una frecuencia cardíaca menor a $50 \mathrm{lpm}$ en caso de encontrarse despierto y menor a $45 \mathrm{lpm}$ si se encuentra dormido. Se incluyen también criterios en relación con trastornos de presión arterial. Estos son presión arterial sistólica por debajo de 90 $\mathrm{mm} / \mathrm{hg}$ y disminución de la presión arterial por ortostatismo. Además, La hipotermia por debajo de 35.6 grados Celsius es un criterio asociado. Por otro lado, pacientes con un peso $75 \%$ menor al promedio para su edad o con un porcentaje de grasa corporal menor al $10 \%$ también cumplen criterios de hospitalización (8). Dentro de estos criterios se incorporan manifestaciones clínicas de trastorno metabólico severo (14). Estas manifestaciones son: síncope, hematemesis, hiperemésis, desequilibrio hidroelectrolítico, pérdida de peso aguda e incluso pacientes con falla en el tratamiento ambulatorio (8).

Durante la estancia hospitalaria, primero se estabilizan los signos vitales de la persona y se determina que no exista otra causa que explique el cuadro del paciente como puede ser diabetes, enfermedad celíaca, enfermedad intestinal inflamatoria o cáncer. Durante esta etapa el paciente se encuentra totalmente encamado por riesgo de lesiones adicionales (14).

Después de estabilizar los signos vitales se avanza al siguiente nivel, en el que se manejará el trastorno metabólico que provocó el internamiento. Inicialmente el individuo tiene permitido movilizarse en silla de ruedas en espacios reducidos. Al avanzar su recuperación se le permitirá ponerse en pie y movilizarse por sus propios medios dentro del salón. Si no presenta ninguna dificultad al hacerlo y sus signos vitales se mantienen dentro de rangos normales, puede ser dado de alta, pero debe continuar un estricto control ambulatorio para mantener el proceso de recuperación total (14). La hospitalización de un paciente con anorexia

\section{(ㄷ) $(1) \Theta \theta$}


es un tema complejo, ya que, a pesar de existir una serie de criterios definidos para valorar la necesidad de internamiento médico, el paciente debe ser abordado no solo desde un punto de vista médico sino también psicológico y nutricional. En la mayoría de casos la recuperación desde el punto de vista médico sucede antes que la psicológica o psiquiátrica, por lo cual es importante resaltar la importancia de continuar un control integral posterior al egreso hospitalario (14).

\section{ETIOLOGÍA}

Este trastorno de la conducta alimentaria incluye factores tanto genéticos como ambientales y el componente cultural y social se encuentran dentro de las causas de mayor relevancia. La anorexia es un trastorno característico del occidente y ha mostrado un incremento importante en las últimas décadas. Dentro de las consideraciones de su aumento están los marcados estándares de belleza que se imponen a las mujeres jóvenes en edades entre los 15 y 25 años, siendo esto atribuido en mayor medida a los medios de comunicación, quienes exigen la delgadez extrema, calificándola como un símbolo de belleza (9).

Entre los elementos que conforman la etiología de los pacientes anoréxicos, se pueden incluir distintos tipos de personalidad como lo son el perfeccionismo y narcisismo junto con rasgos obsesivos compulsivos (9).

Según Siegfried et al, la genética tiene una influencia del $59 \%$, presentando un polimorfismo en el gen que transcribe el factor neurotrófico derivado del cerebro, el cual tiene como parte de su función la plasticidad cerebral, regulación y desarrollo neuronal, explicando así la dificultad para lograr un tratamiento apto (9).

\section{DIAGNÓSTICO DIFERENCIAL}

Algunos autores aseguran que no existe diagnóstico diferencial propiamente dicho y que basta con que el médico realice una buena historia clínica y escuche al paciente y a su familia para realizar el adecuado diagnóstico. Sin embargo, siempre se deben tener en cuenta diferentes patologías que se pueden confundir con anorexia como lo son la enfermedad inflamatoria intestinal, úlcera péptica, hipertiroidismo, diabetes tipo 1 , entre otras (2) (13).

Por otro lado, desde el punto de vista psiquiátrico, debe distinguirse entre anorexia nerviosa, en donde la anorexia es la parte principal del cuadro clínico y la anorexia como consecuencia de otra enfermedad psiquiátrica. La ansiedad, depresión y el trastorno por rumiación pueden causar una importante pérdida de peso en el individuo, pero el miedo intenso a la ganancia ponderal y las preocupaciones excesivas sobre su propio peso no están presentes (2) (13).

\section{CLÍNICA}

El diagnóstico de la anorexia nerviosa es principalmente clínico, por lo que se deben conocer muy bien sus características. Típicamente se necesita la presencia de la tríada sintomática conocida como "las $3 \mathrm{~A}$ ": amenorrea, anorexia y adelgazamiento durante un periodo de 3 - 6 meses, luego de haber iniciado un régimen de pérdida de peso. Generalmente existe algún factor desencadenante de los síntomas como por ejemplo duelo, ruptura sentimental, mudanza, separación familiar o algún conflicto (2). 
La triada clínica mencionada anteriormente se compone de:

1. Amenorrea: es primaria si la paciente nunca ha menstruado y secundaria si se genera luego de tres períodos, regulares o no. Hace unos años se consideraba que la amenorrea era un hallazgo constante y de hecho lo citaban como necesario para el diagnóstico; sin embargo, actualmente la presencia de esta no es necesaria para realizarlo, puesto que en algunos casos no se encuentra presente por factores como tratamiento con anticonceptivos o presentación de anorexia en el sexo masculino (2) (12).

La amenorrea remite a dos factores etiológicos que son el psicológico y el relacionado con pérdida de peso, hiperactividad física y desnutrición. Es muy importante recalcar que esta persiste luego de la recuperación ponderal, siendo el último síntoma en desaparecer (2).

2. Anorexia: generalmente es la primera manifestación. Se trata de una restricción alimentaria activa no relacionada con la falta o disminución del apetito y se manifiesta como un régimen alimenticio voluntario, cada vez más restrictivo, que puede llegar a la afagia (2).

Este síntoma es sumamente difícil de detectar debido a que el paciente generalmente no lo manifiesta. Al inicio la persona lucha contra el hambre, pero con el pasar del tiempo esta sensación disminuye poco a poco hasta desaparecer y es sustituida por una importante intolerancia a la alimentación. Además, esta manifestación clínica se acompaña de comportamientos peculiares como masticar excesivamente cada bocado de alimento ingerido, comer porciones muy pequeñas y contar las calorías de los alimentos (2).

3. Adelgazamiento: esta manifestación se da como consecuencia de la anorexia sufrida por el paciente. La pérdida de peso puede ser progresiva, pero generalmente supera el $25 \%$ del peso inicial e incluso puede llegar al $50 \%$ del peso normal para la edad. Es muy importante saber que la pérdida ponderal en estas personas produce satisfacción y que los jóvenes anoréxicos tienen una distorsión en la imagen corporal, conocida como trastorno dismórfico corporal, que puede ser generalizada - limitada a ciertas zonas como caderas, mejillas, abdomen, entre otros. Es por esto que, frecuentemente llegan a una grave desnutrición y disminución en los rendimientos intelectuales y físicos antes de que reconozcan la realidad de su trastorno y acepten ayuda (2).

En cuanto a la exploración física el aspecto es bastante orientador, debido a que en estos pacientes se puede evidenciar un cuerpo anguloso, demacrado, rostro cadavérico con ojos y mejillas hundidas, caída de cabello, cabello seco, uñas estriadas y quebradizas, lanugo, piel seca, hipertricosis, palidez, desaparición de características femeninas como mamas y caderas, entre otros (2) (8).

Por otro lado, existen diversas características como aislamiento social, necesidad de dominio expresado a través de su cuerpo, relaciones de dependencia, sexualidad rechazada y suprimida. Estas pueden ser detectadas realizando una historia clínica detallada y ejecutando preguntas como: ¿Cuál fue su mayor y menor peso?, ¿Cuánto considera que debería pesar en este momento?, ¿Cómo se encuentra su ánimo?, ¿Qué ha comido en las últimas 24 horas?, ¿Cuenta calorías o carbohidratos?, ¿Se siente culpable cuando come?, ¿Ha utilizado alguna droga?, ¿Existen trastornos psiquiátricos en su familia? (2) (8).

\section{COMPLICACIONES:}

En la anorexia, el cuerpo debe adaptarse al 
estado de inanición y es por esto que disminuye los requerimientos de energía y el metabolismo al mínimo posible. En consecuencia, se desarrollan múltiples dificultades que tienen repercusiones en la salud y calidad de vida de estas personas, dando lugar a más del $50 \%$ de muertes por esta patología y de hecho se sabe que el rango de muerte es $10-12$ veces mayor que en población sana (8) (15).

Las consecuencias negativas en esta patología se desarrollan debido a la malnutrición, pérdida ponderal y trastornos hidroelectrolíticos originados cuando los individuos se purgan. La severa restricción en la ingesta de alimentos que se autoimpone el sujeto, origina atrofia en diferentes tejidos como por ejemplo músculos, corazón, cerebro y riñones, empeorando la condición a medida que aumenta la pérdida de peso (15).

Algunas de las complicaciones originadas por la anorexia son las siguientes:

1.Síndrome de realimentación: Es un complejo cuadro clínico que engloba múltiples disfunciones orgánicas que ocurren como consecuencia de la nutrición por vía parenteral, oral o enteral en pacientes con subnutrición y se encuentra relacionado con la incapacidad del organismo para producir una adecuada cantidad de adenosín trifosfato (ATP) (8) (16).

El síndrome de realimentación puede conducir a distintas alteraciones como retención de agua y sodio, que lleva a edemas y problemas cardíacos, problemas en el metabolismo de carbohidratos y lípidos, manifestaciones psiquiátricas, déficit de tiamina, hipomagnesemia, hipopotasemia, hipofosfatemia relacionada con problemas cardíacos, disfunción respiratoria, hematológica y neuromuscular (16).

Esta es una condición evitable pero altamente mortal en pacientes con desnutrición severa que se encuentran en tratamiento nutricional y para evitarlo se debe controlar la ingesta energética de la persona y las alteraciones hidroelectrolíticas (16).

2. Cardiovasculares: hasta el $80 \%$ de los adolescentes con anorexia llegan a desarrollar algún tipo de complicación cardiovascular como arritmias, hipotensión, derrame pericárdico, prolapso de la válvula mitral, disminución del gasto cardíaco y en el tamaño del ventrículo izquierdo que puede llevar a bradicardia y disfunción sistólica (11) (15) (17).

3.Óseas: esta enfermedad se acompaña de osteopenia en más del $90 \%$ y osteoporosis en el $30-40 \%$ de las mujeres, todo esto como efecto del hipogonadismo, hipoandrogenismo, hipoestrogenismo, hipercortisolismo, déficit de calcio y vitamina D. El grado de osteoporosis es peso y amenorrea dependiente. Se sabe que la falta de menstruación por más de seis meses y un IMC menor a $15 \mathrm{~kg} / \mathrm{m} 2$ son factores que disminuyen la densidad mineral ósea (15).

4.Metabólicas y endocrinas: la principal complicación es la amenorrea secundaria debido al poco estímulo sobre la secreción de gonadotrofinas. Esta condición se puede generar con una pérdida ponderal del $10-15 \%$ pero en el $20 \%$ de los casos la ausencia de menstruación puede preceder a la pérdida de peso (15).

En esta misma línea, estos enfermos pueden presentar hipoglicemias relacionadas a la ínfima ingesta dietética, reducción en los depósitos de glucógeno, ejercicio excesivo y falta de sustratos para la glucogénesis. La hipoglicemia persistente es un marcador de disfunción hepática y constituye un mal pronóstico (15).

Por otro lado, la excreción de $\mathrm{ADH}$ puede alterarse, produciendo hiponatremia o diabetes insípida parcial e hipernatremia. También, con el 
fin de disminuir el gasto metabólico, puede haber una reducción en la transformación de tiroxina (T4) en triyodotironina (T3) y en una malnutrición severa puede los niveles de T4. Sin embargo, no se encuentra indicada la suplementación con hormonas tiroideas (15).

5. Renales: se puede desarrollar deshidratación, lesión renal por restricción hídrica o vómitos y perder la capacidad de concentración renal que resultaría en una elevación del gasto urinario (17).

6. Dermatológicas: en estos individuos se puede encontrar lanugo, resequedad en piel, prurito relacionado con la inanición e inclusive carotenodermia (11).

7.Gastrointestinales: se produce un retraso en el vaciamiento gástrico y en consecuencia se produce estreñimiento, reflujo gastroesfágico, dolor abdominal y sensación de llenura (17).

8.Carencia de vitaminas y minerales: son frecuentes en los trastornos de la conducta alimentaria. Los déficits más comunes son los de hierro, vitamina $D$ y calcio, pero también puede haber una depleción de zinc y tiamina (17).

\section{TRATAMIENTO:}

El tratamiento ya sea ambulatorio o no, siempre requiere manejo por parte de un equipo multidisciplinario conformado principalmente por médicos, nutricionistas y psicólogos entrenados en el manejo de trastornos de la conducta alimentaria. Estos profesionales brindarán un manejo en donde se incluyen terapias psicológicas, medicamentos y planes nutricionales, siempre adecuándose a las necesidades de cada paciente (3) (8) (10) (15) (18) (19). El seguimiento de esta patología puede darse a nivel hospitalario o ambulatorio, siendo este último el principal, ya que cuando un paciente se interna es porque fracasó la terapia ambulatoria o la enfermedad llegó a ser muy grave (19).

1.Farmacológico: No existen medicamentos aprobados por la FDA para el tratamiento de este trastorno alimenticio, por lo tanto, el uso de fármacos en anorexia nerviosa es experimental. Su utilización debe ser considerada únicamente para el manejo de la agitación aguda en el paciente psiquiátrico, utilizando dosis muy bajas, ya que por lo general estos pacientes son medicamente inestables. Su uso debe suspenderse posteriormente para así evitar posibles efectos secundarios (20).

Los antipsicóticos son algunas de las drogas que pueden utilizarse en esta enfermedad y tienen como objetivo disminuir pensamientos obsesivos, ansiedad severa y mejorar la ganancia de peso (13). Por otro lado, Attia et al. Realizó un estudio durante 16 semanas sobre los efectos producidos en la utilización de la Olanzapina versus placebo. Estos autores encontraron evidencia de que el empleo de este fármaco asoció un incremento estadístico en el índice de masa corporal, por lo tanto se cree que este medicamento tiene efectos positivos y complementarios en cuanto a la ganancia de peso (18). Por último, los inhibidores selectivos de la recaptación de serotonina no tienen evidencia de beneficio para el tratamiento de los síntomas o la prevención de recaídas (13).

2.Psicológico: La terapia basada en la familia (TBF) es la que muestra mayor evidencia para el manejo de anorexia nerviosa en adolescentes menores de 19 años que han tenido la enfermedad durante al menos 3 años y se reconoce como la primera línea terapéutica a nivel internacional (13) (20). Al finalizar el proceso, se estima que entre el $50-70 \%$ de los

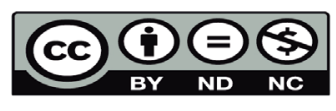


enfermos habrá regresado a un peso saludable y las tasas de recaída por lo general son bajas. La terapia intensiva multifamiliar durante 5 días ha presentado resultados positivos, siendo útil cuando la terapia familiar es insuficiente por sí sola (13).

La TBF consta en la educación, soporte emocional y autorización a los padres para abordar cualquier necesidad que se presente durante el tratamiento por medio de distintas técnicas, estableciendo objetivos específicos para el cambio del comportamiento y disminuyendo conductas de negociación así como minimizando respuestas de tipo emocional o protestas (20).

La terapia basada en la familia se compone de tres fases, en donde se incluyen de 10 a 20 sesiones durante un periodo de seis meses a un año. La primera fase se centra en instruir a los padres para que puedan hacerse cargo, cambiar los comportamientos del paciente e iniciar el aumento de peso bajo supervisión. Esta fase suele extenderse por aproximadamente 3 meses. Cuando la mayoría del peso adecuado para la edad se ha restaurado, se inicia la fase dos con una duración de 2 -3 meses. En esta se incluye el auto control en la alimentación y el inicio de actividad física. Es de suma importancia que en esta etapa el sujeto demuestre dominio sobre estos comportamientos. Finalmente se avanza a la fase 3, que inicia cuando el enfermo logra alimentarse con las cantidades adecuadas para mantenerse saludable sin que los encargados tengan que intervenir. Usualmente esta última etapa se prolonga por 1 a 2 meses (20).

3.Nutricional: los objetivos en la rehabilitación de pacientes anoréxicos son la recuperación del estado nutricional y la mejoría en el peso, para así mejorar las disfunciones desencadenadas por dicha enfermedad. El tratamiento dietético además, permite que el individuo alcance el nivel cognitivo necesario para que pueda comprender y responder de forma adecuada a las intervenciones psicológicas (9) (15).

En el tratamiento nutricional ambulatorio debe tomarse en consideración los siguientes enunciados:

A. Evaluación nutricional: se debe medir la talla y peso, calcular el IMC, tomar los pliegues cutáneos con un lipocalibrador, calcular la circunferencia muscular del brazo y evaluar el índice de cintura - cadera para controlar la distribución de grasa en el paciente. También, se utiliza la impedancia bioeléctrica (BIA) para el estudio de la composición corporal al pie de la cama. Sin embargo, puede ser un método poco fiable porque se altera cuando surgen cambios en la hidratación del individuo y en personas con un IMC menor a 15 kg/m2 (15).

Por otrolado, en estas personas se deben asociar al menos dos métodos de evaluación como por ejemplo el IMC y la impedancia bioeléctrica, para así poder observar los cambios durante el seguimiento. Es importante recordar que en estos pacientes se tiene que calcular el gasto energético en reposo, siendo más adecuado medirlo con calorimetría indirecta, ya que fórmulas como la de Harris - Benedict tienden a infravalorar las necesidades energéticas de pacientes desnutridos (15).

B.Valoración funcional: se considera una herramienta útil para predecir la evolución clínica en estos individuos. Se realiza mediante cuestionarios de riesgo nutricional y evaluación de función muscular, así como dinamometría de la mano con medición en $\mathrm{kg}$ de la fuerza de prensión que se relaciona con la masa libre de grasa corporal o también se pueden utilizar pruebas como la espirometría incentivada y la medición del flujo espiratorio máximo (15).

C. Distribución de macronutrientes: se recomiendaqueel plannutricionalseabalanceado 
y que incluya todos los macronutrientes, dando prioridad a los lípidos y calculándolos al $50 \%$ del total de la dieta. En cuanto a las proteínas debe brindarse entre $1,2-1,5 \mathrm{~g} / \mathrm{kg}$ de peso corporal y el resto corresponderá al aporte de carbohidratos (16).

D.Requerimiento energético: se inicia una dieta con bajo aporte calórico, para así evitar el síndrome de realimentación. Sin embargo, algunos estudios afirman que no se recomienda una dieta muy hipocalórica, ya que este síndrome se asocia al bajo IMC y no a la rápida introducción energética (19).

El cálculo del requerimiento energético se realiza con el peso real del paciente para no sobreestimar el cálculo y elevar el riesgo de síndrome de realimentación. En personas con desnutrición grave, se aconseja brindar entre 25 - $30 \mathrm{kcal} / \mathrm{kg} / \mathrm{día}$, evitando calcular más de $1000 \mathrm{kcal} / \mathrm{día}$ al inicio. En el transcurso de siete días, se debe aumentar en 500 kcal por día para conseguir una ganancia de medio kilogramo (15).

Por otro lado, durante la fase de recuperación de peso, es ideal un aporte calórico de 70 - 100 $\mathrm{kcal} / \mathrm{kg} / \mathrm{día}$, garantizando que se disponga de los nutrientes y energía necesarios en la dieta de cada persona. Además, se sabe que las personas con esta patología necesitan un mayor aporte calórico diario que los sujetos sanos, por lo que se tiene que mantener el exceso de energía por un periodo de tiempo prolongado y luego de 6 meses disminuir a los requerimientos de una persona sana (15).

E. Líquidos: se restringe al máximo el aporte de fluidos, manteniendo siempre el adecuado funcionamiento de los riñones. El aporte hídrico se modificará de acuerdo con la ganancia ponderal del sujeto y con su estado de hidratación. Es importante recordar que el aumento de peso mayor a $1 \mathrm{~kg}$ por semana generalmente se asocia con sobrecarga de líquido que lleva a la retención del mismo (16).

F.Peso: uno de los objetivos es alcanzar el anabolismo mediante la ganancia de peso, alcanzando una ganancia ponderal mínima mayor al 85 - 90\% del peso ideal o un IMC mayor a $18 \mathrm{~kg} / \mathrm{m} 2$ en adultos; mientras que en niños y adolescentes se ha sugerido como razonable un percentil de IMC según sexo y edad entre 13 y 30 . Todo lo anterior tomando siempre en cuenta la historia de peso familiar (15).

Se considera razonable que las personas anoréxicas aumenten de 0,5 - $1 \mathrm{~kg}$ por semana en el contexto ambulatorio y esto puede conseguirse mediante el cálculo de 3500 a 7000 kcal extra semanales, adecuándose a las necesidades de cada individuo (15) (16).

\section{Conclusiones:}

Los trastornos de conducta alimentaria son enfermedades psiquiátricas frecuentes de origen multifactorial que se presentan mayormente durante la adolescencia. La anorexia es una de las enfermedades pertenecientes a este grupo de patologías. En las últimas décadas ha mostrado un importante aumento en el número de casos reportados, en gran parte influenciados por los medios de comunicación, quienes imponen altos estándares de belleza y exigen la delgadez extrema.

La anorexia nerviosa puede desencadenar graves complicaciones somáticas, psiquiátricas o sociales, que incrementan el riesgo de mortalidad en esta población. Es por esto que se requiere una detección temprana y eficaz de este trastorno mediante la utilización de diversas herramientas como historia clínica, examen físico, cuestionarios, entre otros. Una vez diagnosticada se necesita el abordaje por

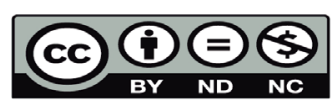


parte de un equipo multidisciplinario, para brindar untratamientoambulatorioointrahospitalarioyasí evitar o mitigar las dificultades desencadenadas por esta enfermedad. Los pacientes que son dados de alta requieren un importante apoyo familiar, monitoreo constante y estricto de sus conductas alimentarias y régimen de actividad física para así disminuir el riesgo de recaídas. Barrueco, M. Melnik, T. Effectiveness of psycosocial interventions in eating disorders: an overview of Cochrane systematic reviews. Einstein. 2015 mayo; 14(2).

\section{BIBLIOGRAFÍA}

1. Chaulet, S. Riquin, É. Avarello, G. Malka, J. Duverger, P. Trastornos de conducta alimentaria en el adolescente. EMC-Pediatría. 2015 setiembre; 50(3).

2.

Treasure J. Antunes, T. Schmidt, U. Eating disorders. The Lancet. 2020 marzo; 395.

Lutter M. Emerging treatments in eating disorders. The Journal of the American Society for Experimental Neurotherapeutics. 2017 mayo; 14.

3. Stice E. Johnson, S. Turgon, R. Eating disorders prevention. Elsevier. 2019 junio; 42(2).

Gorrell S. Murray, S. Eating disorders in males. Elsevier. 2019 octubre; 28(4).

4.

Himmerich H. Treasure, J. Psycopharmacological advances in eating disorders. Taylor \& Francis

5. Group. 2017 setiembre; 11(1).

6. Rome E. Strandjord, S. Eating disorders. Pediatrics in Review. 2016 agosto; 37(8).

Eixea E. Caballer, A. Trabajo final de grado: Revisión bibliográfica sobre los principales

7. tratamientos en la anorexia nerviosa. Repositori Universitat Jaume I. 2016 junio.

8. Hamdan J. Mela, A. Pérez, M. Bastidas, B. Trastornos de la alimentación: Anorexia nerviosa y bulimia nerviosa. RFS Revista Facultad de Salud. 2017 mayo; 9(1).

9. Sangvai D. Eating disorders in the primary care setting. Elsevier. 2016 junio; 43(2).

Erzegovesi S. Bellodi, L. Eating disorders. Cambridge University Press. 2016 junio; 21(4). 10.

Mairs R. Nicholls, D. Assessment and treatment of eating disorders in children and adolescents.

11. 2016 junio; 101(11).

12. Derenne J. The role of higher levels of care for eating disorders in youth. Elsevier. 2019 octubre; 28(4).

13. Gómez C. Palma, S. Miján, A. Rodríguez, P. Matía, P. Loría, V. et al. Consenso sobre la evaluación y el tratamiento nutricional de los trastornos de la conducta alimentaria: Anorexia nerviosa. Nutrición Hospitalaria. 2018; 35(1). 
14. Díaz N. Abordaje dietético-nutricional en la anorexia nerviosa: Prevención del síndrome de realimentación. Instituto de Ciencias de la Conducta Dr Jáuregui S.C.P. 2016 mayo.

16. Peebles R. Hayley, E. Medical complications of eating disorders in youth. Elsevier. 2019 octubre; 28(4).

17. Davis L. Attia, E. Recent advances in therapies for eating disorders [version 1; peer review: 2 approved]. F1000Research. 2019 setiembre.

18. Delgado, P. Jimeno, N. Aspectos nutricionales de la anorexia nerviosa. UvaDoc. [Online].; 2017 [cited 2020 junio 28]. Available from: http://uvadoc.uva.es/handle/10324/24939

19. Lock J. Updates on treatments for adolescent anorexia nervosa. Elsevier. 2019 octubre; 28(4).

20. Englebert, J. Follet, V. Valentiny, C. Anorexia nervosa and first-person perspective: Altruism, family system, and body experience. Psychopathology. 2017 diciembre; 51(1).

21. Brewer, R. Cook, R. Bird, G. Alexithymia: a general deficit of interoception. Royal Society Open Science. 2016 noviembre; 3(10). 